\title{
Is the Contextuality Loophole Fatal for the Derivation of Bell Inequalities?
}

\author{
T.M. Nieuwenhuizen
}

Received: 8 September 2009 / Accepted: 20 April 2010 / Published online: 11 May 2010

(C) The Author(s) 2010. This article is published with open access at Springerlink.com

\begin{abstract}
It is explained on a physical basis how absence of contextuality allows Bell inequalities to be violated, without bringing an implication on locality or realism. Hereto we connect first to the local realistic theory Stochastic Electrodynamics, and then put the argument more broadly. Thus even if Bell Inequality Violation is demonstrated beyond reasonable doubt, it will have no say on local realism, because absence of contextuality prevents the Bell inequalities to be derived from local realistic models.
\end{abstract}

Keywords Bell inequalities $\cdot$ Loopholes $\cdot$ Contextuality $\cdot$ Stochastic electrodynamics

\section{Introduction}

Quantum theory describes in my view the statistics of outcomes of measurements done on an underlying reality known to us as "Nature" or "World", while quantum mechanics or quantum field theory should be called "a theory", or, better, "our best present theory". In my view, in Nature particles are definite entities, subject to certain waves, that partly manifest themselves as the mysterious "quantum fluctuations". This view arose from studying the dynamics of quantum measurements [1], and the subsequent question of what is going on in an individual quantum measurement. Some specific process must be going on in every individual measurement. We have no theory for that at present, but clearly Nature is using it all the time. Quantum theory gives some admittedly strong, but incomplete information about outcomes of experiments.

T.M. Nieuwenhuizen ( $\varangle$ )

Institute for Theoretical Physics, Valckenierstraat 65, 1018 XE Amsterdam, The Netherlands

e-mail: nieuwenh@science.uva.nl 
One may also wonder what is going on in reality with cosmic rays, particles that have traveled to us for millions of years, or, even more stunning, with cosmic microwave background radiation, that traveled more than 13 billion years through basically empty space. These are not questions that should be answered within quantum mechanics, with answers like "In Hilbert space the state of a particle is represented by a state vector...", no, they are questions about what occurs in Nature. I see no other possibility than to assign a reality to cosmic ray particles and to photons, say "balls" or, preferably, "solitons", that traveled all these years through space to us.

"Quantum fluctuations" present a notion taught in every quantum mechanics course without anybody clearly explaining what is fluctuating where. Quantum Mechanics and quantum field theory have the amazing property that we do not have to know these details if our aim is restricted to getting statistical predictions. This situation is somewhat reminiscent to the fact that, given a certain country, we don't have to speak its language to understand the statistics of its population such as the average age, average height, average income, and so on. But one cannot claim to understand the people without knowing their language and their culture. In other words: statistical understanding (quantum theory) is partial understanding that cannot be taken for the full truth as it leaves many subtle questions unanswered.

Stochastic Electromagnetism (SED) [2] is to this date the most promising option to deal with the underlying level of reality. In that theory, "quantum fluctuations" are physical fluctuations of the classical electromagnetic field with a zero-point spectrum. Planck's constant enters by the strength of these fluctuations. A connection with quantum mechanics has been put forward by Cetto and de la Peña already some 15 years ago in their approach called "Linear Stochastic Electromagnetics", see e.g. $[2,3]$. Whether or not this theory is the right direction towards subquantum mechanics is not relevant for the present discussion. Another promising direction is 't Hooft's approach towards entangled quantum states in a local deterministic theory [4].

With quantum fluctuations expressed by SED or a comparable theory, and particles being balls or solitons, the underlying quasi-deterministic level may be called "Stochastic Soliton Mechanics", a name I coined earlier [5]. Double slit interference should then emerge from solitons going through one of the slits and interfering with "idler waves" originating from the other slit. Fluid mechanical interference effects between drops made of the same fluid and waves of this fluid have indeed been observed in the laboratory $[6,7]$.

In his opening address of the 2008 Växjö conference Foundations of Probability and Physics-5, Andrei Khrennikov took the position that violations of Bell inequalities [8] occur in Nature, but do not rule out local realism, due to lack of contextuality: the measurements needed to test Bell inequalities (BI) such as the BCHSH inequality cannot be performed simultaneously [9]. Therefore Kolmogorian probability theory starts and ends with having different probability spaces, and Bell inequality violation (BIV) just proves that there cannot be a reduction to one common probability space. This finally implies that no conclusion can be drawn on local realism, since incompatible information can not be used to draw any conclusion. As explained below, the different pieces of the CHSH inequality involve fundamentally different distribution functions of the hidden variables, which cannot be put together in one over all covering distribution of all hidden variables of the set of considered experiments. To 
our knowledge, the first remarks related to contextuality were made by Cetto, Brody and de la Peña [10]. The contextuality problem was first pointed at in mathematical rigor by Luigi Accardi [11], and then taken by an increasing group of people, e.g. Fine [12], Pitowsky [13], Rastal [14], Kupczynski [15], Garola and Solombrino [16], Khrennikov [17], Volovich [18], Hess and Philipp [19], Sozzo [20] and Zhao, de Raedt and Michielsen [21]. I now also subscribe to this position.

Related works are Accardi's discussion of the chameleon effect, essentially about the role of measurement devices [22] and Adenier's series of works on a deep analysis of the role of detectors [23-25]. A pedagogical discussion of Boole's logics and its role in Bell inequality violation in a daily life setup was presented by Hess et al. [26]. A fundamental analysis of the role of contextuality was performed in monographs by Khrennikov [27, 28]. Finally, we mention that macroscopic, classical entanglement has been demonstrated in the simple setup of two classical Brownian particles [29], a finding which is also relevant to contextual considerations.

At the University of Amsterdam I supervise bachelor projects on Bell inequalities. Students are happy to get insight in the possible structure of the physics behind the quantum formalism. Non-contextuality is a standard ingredient. Let us see how it comes in.

\section{How the Contextuality Enters the Bell Argument}

In the Clauser-Horne-Shimony-Holt (CHSH) setup, one may consider a source that emits pairs of spin 1/2 particles, one going to station A "Alice" and the other, in opposite direction, to station B "Bob". At each station one out of two possible measurements is performed, $A_{1}$ or $A_{2}$ by Alice and $B_{1}$ or $B_{2}$ by Bob. In case $A_{1}$ the particles spin is measured along the axis in direction $\mathbf{a}_{1}$ and in case $A_{2}$ along the axis in direction $\mathbf{a}_{2}$. Likewise $B_{1,2}$ corresponds to measurements along axis $\mathbf{b}_{1,2}$ respectively. The outcomes, "up" or "down" along axis $\mathbf{a}_{i}$ for A are denoted as $S_{A_{i}}= \pm 1$, respectively, and likewise for the measurement by B along axis $\mathbf{b}_{j}$ as $S_{B_{j}}= \pm 1$. The measurement is repeated many times. Ideally - if all particles of all pairs are measured - the recordings on the two detectors come as pairs. In each case, the direction of the axis is known and it is recorded whether "up" or "down" was measured along the chosen axis. Putting afterwards the results from both detectors together, one determines the four correlators $C_{i j}=\left\langle S_{A_{i}} S_{B_{j}}\right\rangle$ for $i=1,2$ and $j=1,2$ by averaging the outcomes over the pairs. From these four objects one makes the combination proposed by Clauser, Horne, Shimony and Holt (CHSH) [30],

$$
\mathrm{BCHSH} \equiv C_{11}+C_{12}-C_{21}+C_{22},
$$

where "B" stands for Bell. Since only \pm 1 variables are involved, it will clearly hold that each $\left|C_{i j}\right| \leq 1$ and BCSHS $\leq 4$. But a stronger bound can be derived. Manipulating with ingredients inside the averages, one has

$$
\mathrm{BCHSH}=\left\langle\left(S_{A_{1}}-S_{A_{2}}\right) S_{B_{1}}+\left(S_{A_{1}}+S_{A_{2}}\right) S_{B_{2}}\right\rangle .
$$


Because the $S$ variables are all \pm 1 , one of the two combinations will be zero, while the other is \pm 2 . This implies a version of the Bell inequalities,

\section{$\mathrm{BCHSH} \leq 2$.}

There are many papers that investigate in great rigor the validity of the steps made here, and they again lead to this result.

In the quantum mechanical description of the measurement, the state is supposed to be pure and described by the singlet state vector $|\psi\rangle=\left(\left|\uparrow_{A}\right\rangle\left|\uparrow_{B}\right\rangle-\right.$ $\left.\left|\downarrow_{A}\right\rangle\left|\downarrow_{B}\right\rangle\right) / \sqrt{2}$. The measurement of the particle spin along axis $\mathbf{a}_{i}$ is described by the operator $\mathbf{a}_{i} \cdot \vec{\sigma}$, where $\vec{\sigma}=\left(\sigma_{x}, \sigma_{y}, \sigma_{z}\right)$ are the three Pauli matrices with $\sigma_{z}=\operatorname{diag}(1,-1)$ and we omit the prefactor $\frac{1}{2} \hbar$. Carrying out the manipulations, one finds that the maximum over the possible directions is taken when all vectors are in a plane, $\mathbf{a}_{1}$ is perpendicular to $\mathbf{a}_{2}$ and $\mathbf{b}_{1}$ perpendicular to $\mathbf{b}_{2}$, while the angle between $\mathbf{a}_{1}$ and $\mathbf{b}_{1}$ is $45^{\circ}$. The value is then BCSHS $=2 \sqrt{2}$ [30]. In particular, a value $2<$ BCSHS $<2 \sqrt{2}$ is allowed by quantum mechanics, but violates the Bell inequality (3) and is therefore often believed to rule out local realism.

We have not discussed how exactly the measurement is carried out, only that the results of all pairs were put together. It is easy to just fix $i$ and $j$, say $i=1, j=2$ and then to collect enough measurement outcomes to allow a good statistical analysis. This is how one uses, say, a neutron beam, during, say, 30 minutes. Next, in a standard setup one changes either $i$ or $j$, and repeats the measurement during, say, another 30 minutes. In this way, the four correlators $C_{i j}$ are determined from consecutive sets of measurements. This setup is good enough to get their values and to show that the Bell inequality (3) can be violated. It was applied in the first test of BIV by Freedman and Clauser [31].

Bell, however, proposed to choose the measuring directions at A and B randomly from their two possibilities, at a moment well after the particles left the source, but well before they arrive at A and B. It is this selection procedure that brings in the issue of locality into the problem, that is to say, the question whether all speeds are less than the speed of light; if not, then the situation is called non-local. Now if the particles are separated from each other more than their travel time multiplied by the speed of light, and detector directions are randomly chosen, but happen to be in parallel directions, then it appears always that one of them gives an "up" registration and the other a "down" registration. From the point of angular momentum conservation this is obvious, but it is not obvious how the particles "get this done". Indeed, if the information about the-in this case parallel-directions of the measurement axes is know only when the particle distance is larger than $c t$, then, it seems, this information has to be transmitted between the particles with a speed larger than $c$. For this reason, Bell's conclusion is that BIV may point at non-locality. Alternatively, he noticed, it may be due to the fact that registered values are not related to properties the particles had before the measurement, a break of realism. Either one or the other is broken, so BIV proves, according to Bell, a breakdown of local realism. If this is true, it puts a major barrier to simple views on the reality underlying quantum theory and a rather hopeless starting point for attempts to improve on quantum theory by formulating a subquantum theory. Absence of local realism is counterintuitive, not to say awkward, so before giving it up, we should really have not any other option. 
Be it as it may, the first experiment along the lines devised by Bell was carried out by Aspect, Dalibard and Roger in 1982, thus consistent with having ruled out local realism [32]. Their work generated a whole field of research, with many contributions reported in conferences. So far, it is agreed that BIV occurs in many different systems, e.g. for photons, neutrons, ions and kaons.

The contextuality issue arises in this discussion because in definition (1) we have put together correlators that could not be measured simultaneously. In particular, we have identified the averaging in the four terms, even though fundamentally different objects are averaged over. In the standard setup one measures them in separate runs. In the Bell-Aspect setup one randomly chooses the directions of the measurement axes, but by the time the particles arrive, it is set at some choice and from then on it looks as if it had been in that state all the time. So in local models contextuality is also present, and just in the same way.

\section{Hidden Variable Models}

\subsection{Bell's Hidden Variable Description}

Bell considers that the measurements outcomes, the $S_{A_{i}}= \pm 1$ are determined by some set of hidden variables. Let us denote the set pertaining to the measured particles schematically by $\lambda$; being created as a pair, it is supposed that both particles both share the same set $\lambda$, that travels with them. It seems natural to follow Bell and assume

$$
C_{i j}=\int \mathrm{d} \lambda \rho(\lambda) S_{A_{i}}(\lambda) S_{B_{j}}(\lambda)
$$

In this way the four correlators all involve the same $\rho(\lambda)$. Therefore (3) can again be derived from (1) using (2), with angular brackets now denoting integrals over $\rho(\lambda)$. Since measurements hint that values $2<B C H S H<2 \sqrt{2}$ are possible, Bell concludes that local hidden variable models do not work and that Nature lacks local realism.

This argument seems so clear that most in the physics community are convinced that Bell is right.

\subsection{About Loopholes}

Various loopholes are known. The first is the detection loophole-in experiments with photons at most $20 \%$ of them are detected. Such may lead to biases. It was closed in the experiment of Rohe et al. [34]. The second is the locality loophole: in experiments with ions the particles are not well separated, thus not excluding the possibility of information transfer at speeds lower than light. It was closed in the experiment of Weihs et al. [35]. For neutron double slit interferometry the spin information can not even in principal be separated from their path information [36]. Another case is the coincidence loophole: when can we speak about the detection of a pair [37]. Recently attention was payed to the fair sampling loophole. So far, fair sampling is a hidden assumption in the analysis of data, that cannot be checked. G. Adenier has defended 
in his PhD-thesis that BIV proves that the fair sampling assumption is violated, not local realism [38].

Since after 25 years since the Aspect experiment it appears still to be very hard to close all loopholes in a single experiment it has been supposed, see e.g. Santos [39], that Nature resists loophole-free Bell experiments. Still, this all is not our main theme. Our point will be that Bell's argument fails even before the issue of these loopholes has to be addressed, because of there is an even more severe one, the contextuality loophole, that cannot be closed at all.

\subsection{Improved Hidden Variables Description}

Bell's original argument, mentioned above, would not convince Niels Bohr, since the detectors have not been taken into account. Clearly, the detectors consist of many particles and will also have hidden variables, $\lambda_{A_{i}}$ and $\lambda_{B_{j}}$. In this setup, each of the four correlators can be written as

$$
C_{i j}=\int \mathrm{d} \lambda \int \mathrm{d} \lambda_{A_{i}} \int \mathrm{d} \lambda_{B_{j}} \rho_{i j}\left(\lambda, \lambda_{A_{i}}, \lambda_{B_{j}}\right) S_{A_{i}}\left(\lambda, \lambda_{A_{i}}\right) S_{B_{j}}\left(\lambda, \lambda_{B_{j}}\right),
$$

where it is to be noted that we assume the non-signaling condition, id est, that the measured value at A does not involve any parameter of B, hidden or not. (We should stress that for $i=1, j=2$ the subscript 1,2 of $\rho_{12}$ in (5) does not point at an explicit dependence but only reminds us that this distribution depends on $\lambda_{A_{1}}, \lambda_{B_{2}}$. The subscript could be dropped when writing $\rho_{12} \rightarrow \rho\left(\lambda ; \lambda_{A_{1}} ; \cdot ; \cdot \lambda_{B_{2}}\right)$ and likewise for the other three cases. So there is no hidden assumption of non-locality. We thank an unknown referee for drawing attention to this issue.)

To come back to his original steps, Bell assumed that the four $\rho_{i j}$ arise from one global distribution function $\rho_{G}$ [8], so that, for instance,

$$
\rho_{12}\left(\lambda, \lambda_{A_{1}}, \lambda_{B_{2}}\right)=\int \mathrm{d} \lambda_{A_{2}} \int \mathrm{d} \lambda_{B_{1}} \rho_{G}\left(\lambda, \lambda_{A_{1}}, \lambda_{A_{2}}, \lambda_{B_{1}}, \lambda_{B_{2}}\right)
$$

and likewise for $\rho_{11}, \rho_{21}, \rho_{22}$. This is the new way in which contextuality enters: it is assumed that there exists a covering distribution $\rho_{G}$ of the sets of hidden variables of all the measurements, even though they cannot be carried out simultaneously. (In our alternative notation, Bell assumes that a $\rho_{G}$ exists for which $\rho\left(\lambda ; \lambda_{A_{1}} ; \cdot ; \cdot ; \lambda_{B_{2}}\right)=$ $\int \mathrm{d} \lambda_{A_{2}} \int \mathrm{d} \lambda_{B_{1}} \rho_{G}\left(\lambda, \lambda_{A_{1}}, \lambda_{A_{2}}, \lambda_{B_{1}}, \lambda_{B_{2}}\right)$, and similarly for the three other marginals.

With this relation, the manipulations that led from (1) to (3) can be repeated and Bell derived the same inequality as without accounting for detector hidden variables [8]. It being violated in experiment, one then concludes that Bell's simpler argument was right anyhow, the detectors bring no new information, and hidden variable models are excluded. The remaining focus is then to close the loopholes and prove that Bell was indeed right.

\section{Dynamics of Quantum Measurements}

The point at stake is thus the role of the hidden variables of the detectors. Bell did include them explicitly in his considerations, with a swift modification of the argument, 
that led to the same result. Such a swift way to deal with quantum measurements may be blamed to an attitude created by the projection postulate of quantum mechanics. In textbooks it is mostly postulated that a quantum measurement amounts to a nonunitary projection of the quantum state on an eigenstate of the measured operator. This idealized notion is completely different from what is common practice in experimental laboratories. Indeed, every small piece of the experimental setup is believed to be governed by quantum mechanics, and the same should hold for the whole apparatus. In other words, quantum measurements are dynamical processes too that must be described within quantum mechanics.

Indeed, it has been possible to consider a rich enough, solvable model for an apparatus that can perform the measurement of a spin $\frac{1}{2}$. The apparatus is an Ising magnet coupled to a bath, with the magnet consisting of a large number of quantum spins $\frac{1}{2}$, coupled to each other only via their $z$-components (Ising character). The magnet starts out in a metastable paramagnetic state, and by coupling to the tested spin, the magnetization is driven into its stable up- or down ferromagnetic state. Here the metastability offers a multiplication of the weak quantum signal of the tested spin into the macroscopic, stable up- or down value of the magnetization at the end of the measurement. Whether this magnetization ("pointer variable") is observed or not, does not play a role-what was relevant was the physical interaction between tested system and the apparatus. The bath is also needed, namely for dumping the excess (free) energy from the initial state of high (free) energy and for decoherence of the Schrödinger cat states. In this model, the Schrödinger cat states disappear quickly, first by an NMR-type dephasing due to the interaction of the tested spin with the spins of the magnet, and then, in the dephased situation, all memory of the initial state is erased by decoherence due to the coupling to the bath. That it is a two-step process if often overlooked from studies of models that do not have enough relevant physics.

This approach thus describes a quantum measurement as a specific process of quantum mechanics, in which two important timescales are concerned: the small dephasing time together with the somewhat larger decoherence time of the off-diagonal terms, and the larger registration time of the diagonal elements, that is, the time in which the up- or down magnetization is built up. If all are still rather small, one may consider these processes effectively as "instantaneous" and the collapse as a nonunitary evolution. This is what is taught in most textbooks, and we stress that to an extent it is misleading. The collapse view holds only in an effective sense, in reality the complete dynamics is unitary in the full Hilbert space of tested system and the apparatus. From the point of view of the tested system, it is an open system dynamics.

\subsection{Did Bell Make an Additional Assumption?}

The assumption of the existence of a $\rho_{G}$, see (6) is crucial in Bell's derivation of his equalities when including detectors.

But it is absolutely not true that, if one knows the marginals, here the $\rho_{i j}\left(\lambda, \lambda_{A_{i}}, \lambda_{B_{j}}\right)$ for $i=1,2$ and $j=1,2$, one may conclude that a common distribution $\rho_{G}$ exists. There are theorems on this and there are explicit examples in which some probabilities then have to be negative $[19,33]$ or do not even exist because of incompatible requirements to be fulfilled. In either case it is safe to say 
then that a common $\rho_{G}$ does not exist. Physically this is not a complete surprise, because anyhow the relevant experiments could not be carried out simultaneously. This uncomfortable knowledge thus appears to express itself also by absence of a common probability distribution (mathematicians say: absence of a common probability space). In other words, the initial problem of incompatible quantum measurements is not healed in any way, if thrown out of the front door, it re-enters through the backdoor.

So it can be concluded that Bell derived his inequality by additionally assuming the existence of a global distribution of all hidden variables. Violation of the inequality demonstrates the incorrectness of this assumption, without any connection to the physics of local realism.

\section{Stochastic Electrodynamics}

So far, so good, the above is common knowledge- even though not commonly accepted. On my way back from the Växjö 2008 conference Foundations of Probability and Physics-5 to my hometown Amsterdam, when waiting at the airport of Copenhagen - it had to be there-I finished a thought that I actually started a year before, at the same place in the same setting. I realized that a physical argument can be brought into the discussion of hidden variable theories such as SED and alikes. In such theories there are specific hidden-or just uncontrolled-variables, those that, at some initial time, set the stochastic forces acting on the measurement apparatuses. A different setting of an apparatus corresponds to a physically different situation and thus to physically different sets of these hidden variables. In each setting, they drive the quantum working of the relevant apparatus, including opposite outcomes when members of a pair are measured along parallel axes. Because of the different physical setups, there is no reason why for different apparatus directions the hidden variables should have the same nature, that is, have a common distribution, that is, be defined on a common probability space.

This can be made more explicit by imagining that when Alice's detector is in direction $\mathbf{a}_{1}$ (to perform measurement $A_{1}$ ), there will be put some other apparatus in direction $\mathbf{a}_{2}$. As long as it does not disturb the measurement $A_{1}$, it is immaterial what this second apparatus is exactly doing, but for sure it will be driven by the hidden variables that would drive Alice's detector were it in this direction. Now it is clear that we speak about physically exclusive situations, each setup $A_{1,2}$ is distinctive and it excludes the other, $A_{2,1}$ : One can't have the cake and eat it. Again, for this very reason there is no justification to assume that their hidden variables are described by a common distribution $\rho_{G}$. One cannot neglect the physics of the other apparatus, some definite process is happening in it too, that could be specified, but need not be for our argument.

In any hidden variable theory, one may expect emission of radiation by Lorentz damping, i.e. accelerated electrons. This is a physical effect, which in SED is statistically balanced by the stochastic forces to reach an equilibrium "quantum" state, due to the presence of a fluctuation-dissipation theorem [2]. This brings once more a physical aspect of detectors, once more precluding attempts to put different setups 
together. Even if this Lorentz damping is not measurable nowadays, we only need to think of the heating of air by the apparatus (and by the second, irrelevant one, if it is there), surely a measurable effect, to realize that different setups of the detectors exclude each other and thus have no cause for possessing a common hidden variables distribution.

More explicitly, in the case of detector at station $\mathrm{A}$ in the direction $\mathbf{a}_{i}$ and the detector at station $\mathrm{B}$ in direction $\mathbf{b}_{j}$, with $i=1,2$ and $j=1,2$, a local hidden variable theory may assume that there exist hidden variables $\lambda$ for the tested pair and further $\lambda_{A_{1}}, \lambda_{A_{2}}, \lambda_{B_{1}}$ and $\lambda_{B_{2}}$ connected to all possible directions of the detectors, with a distribution $\rho_{i j}\left(\lambda, \lambda_{A_{1}}, \lambda_{A_{2}}, \lambda_{B_{1}}, \lambda_{B_{2}}\right)$, that gives, very different from (6),

$$
\rho_{i j}\left(\lambda, \lambda_{A_{1}}, \lambda_{B_{2}}\right)=\int \mathrm{d} \lambda_{A_{2}} \int \mathrm{d} \lambda_{B_{1}} \rho_{i j}\left(\lambda, \lambda_{A_{1}}, \lambda_{A_{2}}, \lambda_{B_{1}}, \lambda_{B_{2}}\right) .
$$

In case the source emits an electron pair or an electron-positron pair, the SternGerlach apparatuses stand in the directions $\mathbf{a}_{i}$ and $\mathbf{b}_{j}$ for each of the four settings $i=$ $1,2, j=1,2$. The fact that $\rho_{11}\left(\lambda, \lambda_{A_{1}}, \lambda_{A_{2}}, \lambda_{B_{1}}, \lambda_{B_{2}}\right) \neq \rho_{21}\left(\lambda, \lambda_{A_{1}}, \lambda_{A_{2}}, \lambda_{B_{1}}, \lambda_{B_{2}}\right)$ is inherent to the physically different setting of the apparatus at station $A$. The fact that the apparatus used in setting $A_{1}$ needs not have the same specifications as the apparatus used in setting $A_{2}$ underlines this point. Similarly, $\rho_{11} \neq \rho_{12}, \rho_{11} \neq \rho_{22}$, $\rho_{12} \neq \rho_{21}, \rho_{12} \neq \rho_{22}$ and $\rho_{21} \neq \rho_{22}$. Within the no-signaling assumption (2) will read

$$
\begin{aligned}
\mathrm{BCHSH}= & \int \mathrm{d} \lambda \int \mathrm{d} \lambda_{A_{1}} \int \mathrm{d} \lambda_{A_{2}} \int \mathrm{d} \lambda_{B_{1}} \int \mathrm{d} \lambda_{B_{2}} \\
& \times\left\{\left[\rho_{11}\left(\lambda, \lambda_{A_{1}}, \lambda_{A_{2}}, \lambda_{B_{1}}, \lambda_{B_{2}}\right) S_{A_{1}}\left(\lambda, \lambda_{A_{1}}\right)\right.\right. \\
& \left.-\rho_{21}\left(\lambda, \lambda_{A_{1}}, \lambda_{A_{2}}, \lambda_{B_{1}}, \lambda_{B_{2}}\right) S_{A_{2}}\left(\lambda, \lambda_{A_{2}}\right)\right] S_{B_{1}}\left(\lambda, \lambda_{B_{1}}\right) \\
& +\left[\rho_{12}\left(\lambda, \lambda_{A_{1}}, \lambda_{A_{2}}, \lambda_{B_{1}}, \lambda_{B_{2}}\right) S_{A_{1}}\left(\lambda, \lambda_{A_{1}}\right)\right. \\
& \left.\left.+\rho_{22}\left(\lambda, \lambda_{A_{1}}, \lambda_{A_{2}}, \lambda_{B_{1}}, \lambda_{B_{2}}\right) S_{A_{2}}\left(\lambda, \lambda_{A_{2}}\right)\right] S_{B_{2}}\left(\lambda, \lambda_{B_{2}}\right)\right\} .
\end{aligned}
$$

Clearly, with all the $\rho_{i j}$ different, one cannot derive the Bell inequality (3). That it may be violated in practice has no implication, since (3) can only be derived under the additional assumptions that all $\rho_{i j}$ coincide, say they equal some $\rho_{G}$. Thus the contextuality loophole cannot be closed without this additional assumption. If the resulting Bell inequality is violated, the blame is on this assumption, not on local realism.

Within SED there is a clear understanding of the Bell-Aspect switching of detector directions: this just has no influence. What counts is the position of the detector at the moment when the particle arrives, not what happens before. Freedman and Clauser already employed this fact when considering detectors without random switching [31].

David Mermin has formulated a pedagogic model where the members of the particle pairs carry instruction sets for the outcomes of the detectors, which contradicts this conclusion [40]. However, Adenier showed that his model can reproduce quantum results if non-detection events are included in the instruction sets [41]. 


\section{Conclusion}

Violations of Bell inequalities occur in Nature if loophole-free situations can be reached. The BIV of quantum physics are adequately explained by quantum theory. Though loopholes have yet not been closed, we would be very surprised if quantum theory would not give the right answer. Indeed, if it did not, then how could it work so well otherwise, so well that as yet borders of quantum theory are not suspected to be established in any near future?

It has always stunned me that Bell's simple hidden variables argument could have such profound implications as the absence of local reality. With some experience in deriving physical results to explain observations in various fields, the Bell analysis has always appeared rather abstract (mathematical) and suspiciously simple to me. The above concrete step of a physical implementation of the non-contextuality argument makes clear to me on a physical basis that Bell just assumed a not necessarily satisfied mathematical condition. The above contextuality argument puts his conclusion where it should be: a mathematical derivation devoid of a clear physical mechanism, that can be refuted on the basis of an ill motivated mathematical assumption, contextuality, or, as we showed, on the basis of obvious physics, the exclusiveness of different detector setups. This contextuality loophole cannot be closed, at least not without additional assumptions. And if they are made and the resulting Bell inequality is derived but violated in practice, then this assumption is to be blamed, not local realism. In this regard we reach a similar conclusion as Adenier, who analyzed the fair sampling assumption [23-25].

So far, in literature it is claimed only that a violation of Bell inequalities leads to absence of a common distribution. Our physical argument makes clear that it must also be absent when the BI are not violated.

Assuming a common distribution function for hidden variables of incompatible experimental setups looks like comparing apples to oranges. It is know that two apples plus three oranges do not add up to five bananas. Likewise, even when combining the outcomes of results of incompatible setups does lead to results described by quantum theory, this managing of data does not yield information about deep physical properties such as locality or realism. The physical input is much too poor to address those physical questions. They are, in my view, out of sight of the progress in physics that we may hope for in next decades.

Bell inequalities are of profound physical interest, as ever, but they have no say on local reality. Experimental tests of non-local realism, though reported in Ref. [42] in connection with Bell inequalities, are actually far beyond the present level of understanding and manipulation. Nature may possess local realism or not, Bell inequalities have no say on that. For now we can just keep our cards on the familiar assumption of Nature possessing local realism.

As for searching the local reality underlying quantum theory, I conclude that Bell has unfortunately obscured the goal. We shall gratefully forgive him, he asked important questions and his efforts led to new fields such as quantum communication and quantum cryptography. But abstract mathematical reasoning has a faint chance to capture relevant physical mechanisms, and once again this was the lesson to learn. Now it is time to get physics back to the forum of particles, waves, forces and hidden 
variables. With Bell inequalities out of the way, Einstein's dream of a local realistic theory of Nature is as alive as ever. It is really time to move on and make it real!

Open Access This article is distributed under the terms of the Creative Commons Attribution Noncommercial License which permits any noncommercial use, distribution, and reproduction in any medium, provided the original author(s) and source are credited.

\section{References}

1. Allahverdyan, A.E., Balian, R., Nieuwenhuizen, Th.M.: Europhys. Lett. 61, 452 (2003)

2. de la Peña, L., Cetto, A.M.: The Quantum Dice: An Introduction to Stochastic Electrodynamics. Kluwer, Dordrecht (1996)

3. de la Peña, L., Cetto, A.M.: In: Adenier, G., Khrennikov, A.Y., Nieuwenhuizen, T.M. (eds.) Quantum Theory: Reconsideration of Foundations-3. AIP Conference Proceedings, vol. 810, p. 131. AIP, Melville (2006)

4. 't Hooft, G.: arXiv:0908.3408

5. Nieuwenhuizen, T.M.: In: Adenier, G., Khrennikov, A.Y., Nieuwenhuizen, T.M. (eds.) Quantum Theory: Reconsideration of Foundations-3. AIP Conference Proceedings, vol. 810, p. 198. AIP, Melville (2006)

6. Couder, Y., Fort, E.: Phys. Rev. Lett. 97, 154101 (2006)

7. Eddi, A., Fort, E., Moisy, F., Couder, Y.: Phys. Rev. Lett. 102, 240401 (2009)

8. Bell, J.S.: Speakable and Unspeakable in Quantum Mechanics. Cambridge University Press, Cambridge (1987)

9. Khrennikov, A.Y.: arXiv:0709.3909

10. Cetto, A.M., Brody, T., de la Peña, L.: Lett. Nuovo Cimento 5, 177 (1997)

11. Accardi, L.: Phys. Rep. 77, 169 (1981)

12. Fine, A.: Phys. Rev. Lett. 48, 291-295 (1982)

13. Pitowsky, I.: Phys. Rev. Lett. 48, 1299-1302 (1982)

14. Rastal, P.: Found. Phys. 13, 555-575 (1983)

15. Kupczynski, M.: Phys. Lett. A 116, 417-422 (1986)

16. Garola, C., Solombrino, L.: Found. Phys. 26, 1121 (1996)

17. Khrennikov, A.Yu.: Found. Phys. 32, 1159-1174 (2002)

18. Volovich, I.V.: In: Khrennikov, A.Y. (ed.) Proc. Conf. Quantum Theory: Reconsideration of Foundations. Ser. Math. Modeling, vol. 2, p. 423. Växjö University Press, Växjö (2002)

19. Hess, K., Philipp, W.: In: Khrennikov, A.Y. (ed.) Proc. Conf. Foundations of Probability and Physics3. AIP Conference Proceedings, vol. 750, pp. 150-155. AIP, New York (2005)

20. Garola, C., Sozzo, S.: quant-ph/0703260 (2007)

21. Zhao, S., de Raedt, H., Michielsen, K.: Found. Phys. 38, 322 (2008)

22. Accardi, L., Imafuku, K., Regoli, M.: Infin. Dimens. Anal. Quantum Probab. Relat. Top. 51, 1 (2002)

23. Adenier, G.: AIP Conference Proceedings 1101 (2009)

24. Adenier, G.: J. Phys. B 40, 131-141 (2007)

25. Adenier, G.: Am. J. Phys. 76, 147 (2008)

26. Hess, K., Michielsen, K., De Raedt, H.: Europhys. Lett. 87, 60007 (2009)

27. Khrennikov, A.Y.: Interpretations of Probability, 2nd edn. de Gruyter, Berlin (2009)

28. Khrennikov, A.Y.: Contextual Approach to Quantum Formalism. Springer, Berlin (2009)

29. Allahverdyan, A.E., Khrennikov, A., Nieuwenhuizen, T.M.: Phys. Rev. A 72, 032102 (2005)

30. Clauser, J.F., Horne, M.A., Shimony, A., Holt, R.A.: Phys. Rev. Lett. 49, 1804 (1969)

31. Freedman, S.J., Clauser, J.F.: Phys. Rev. Lett. 28, 938 (1972)

32. Aspect, A., Dalibard, J., Roger, G.: Phys. Rev. Lett. 49, 1804 (1982)

33. Vorobev, N.N.: Theory Probab. Appl. 7, 147-162 (1962)

34. Rohe, M.A., Kielpinski, D., Meyer, V., Sackett, C.A., Itano, W.M., Monroe, C., Wineland, D.J.: Nature 409, 791 (2001)

35. Weihs, G., Jennewein, T., Simon, C., Weinfurther, H., Zeilinger, A.: Phys. Rev. Lett. 81, 5039 (1998)

36. Hasegaga, Y., Loidl, R., Barudek, G., Baron, M., Rauch, H.: Nature 425, 45 (2003)

37. Larsson, J.A., Gill, R.D.: Europhys. Lett. 67, 707 (2004) 
38. Adenier, G.: Local realist approach and numerical simulations of nonclassical experiments in quantum mechanics. Thesis, Växjö University, November 2008

39. Santos, E.: Found. Phys. 34, 1643 (2004)

40. Mermin, N.D.: Am. J. Phys. 49, 940 (1981)

41. Adenier, G.: Am. J. Phys. 76, 147 (2008)

42. Gröblacher, S., Paterek, T., Kaltenbaek, R., Brukner, C., Zukowski, M., Aspelmeyer, M., Zeilinger, A.: Nature 446, 871 (2007) 